\title{
Tamm plasmon-polaritons structures with Bi-substituted garnet layers
}

\author{
Tatyana Mikhailova*, Alexander Shaposhnikov, Anatoly Prokopov, Andrey Karavainikov, Sergey Tomilin, \\ Sergey Lyashko, and Vladimir Berzhansky
}

V. I. Vernadsky Crimean Federal University, 295007 Simferopol, Russia

\begin{abstract}
New original Tamm plasmon-polaritons (TPP) structures with Bi-substituted iron garnet and $\mathrm{Au}$ plasmonic layers were proposed, synthesized and investigated. The structures with single and double garnet layers were modelled to form a TPP mode at the center of photonic band gab. The top Au layer has the gradient thickness varied in the range from 0 to $65.2 \mathrm{~nm}$. It was found the features of TPP resonances as a function of the thickness of metal coating. The resonances on TPP have the maximum optical quality factor and transmission at the vicinity of Au thickness of $30 \mathrm{~nm}$. These configurations are optimum to form the highest intensity of electric field of light wave in the area of the magnetic layers. It was found the spectral blue and red shifts of TPP mode with increasing of Au thickness. The blue and red shifts can be explained respectively by structural and thickness changes of $\mathrm{Au}$ coating. The maximum resonant values of Faraday rotation were $-2.1^{\circ}$ at $664 \mathrm{~nm}$ and $-12.3^{\circ}$ at $645 \mathrm{~nm}$ for structures with single and double garnet layers, respectively, and thickness of Au coating of $65.2 \mathrm{~nm}$.
\end{abstract}

\section{Introduction}

The one-dimensional nanostructures of photonic crystals with bismuth-substituted iron garnet (Bi: $\mathrm{IG})$ layers are perspective for magnetooptical (MO) sensors in optoelectronic devices allowed significant rotation of the polarization plane of the transmitted light. One of the first proposed structures in magnetophotonics was the microcavity based on nonmagnetic dielectric Bragg mirrors (BM) and a Bi: IG layer [1-5]. In such structures, Faraday and Kerr rotation enhancement is achieved most effectively. Later, the photonic and magnetophotonic crystals limited on the one side by a metal were investigated [6-10]. The amplification of MO effects in these structures are result of the excitation of the optical Tamm state or Tamm plasmon-polaritons (TPP). In addition the TPP mode can be combined with resonances of another nature [11-15]. In our previous works, we proposed and investigated the new type of microcavity structure on basis of nonmagnetic dielectric $\mathrm{BM}$ and double iron garnet layer with low and high Bi content [15-17]. In this paper, the new effective magnetophotonic structures with TPP resonance mode are proposed and realised.

\section{Experimental technique and modelling}

The investigated TPP structures consist of a seven-pair dielectric BM of titanium and silicon oxides, on the surface of which single or double garnet layer, silicon oxide buffer layer and Au plasmonic coating are placed consistently. The top $\mathrm{Au}$ plasmonic coating has the gradient thickness. Formulas of structures are listed below

No. 1 - GGG / $\left[\mathrm{TiO}_{2} / \mathrm{SiO}_{2}\right]^{7} / \mathrm{M} 1 / \mathrm{SiO}_{2} / \mathrm{Au}$,

No. $2-\mathrm{GGG} /\left[\mathrm{TiO}_{2} / \mathrm{SiO}_{2}\right]^{7} / \mathrm{M} 1 / \mathrm{M} 2 / \mathrm{SiO}_{2} / \mathrm{Au}$.

There GGG is substrate of gadolinium gallium garnet with crystallographic orientation of (111). M1 and M2 are $\mathrm{Bi}$ : IG of compositions of $\mathrm{Bi}_{1.0} \mathrm{Y}_{0.5} \mathrm{Gd}_{1.5} \mathrm{Fe}_{4.2} \mathrm{Al}_{0.8} \mathrm{O}_{12}$ and $\mathrm{Bi}_{1.5} \mathrm{Gd}_{1.5} \mathrm{Fe}_{4.5} \mathrm{Al}_{0.5} \mathrm{O}_{12}$, respectively. Schematic diagrams of configurations are shown in Figure 1. The buffer layer of silicon oxide was used to reduce the absorption of light at the magnetic layer-metal interface.
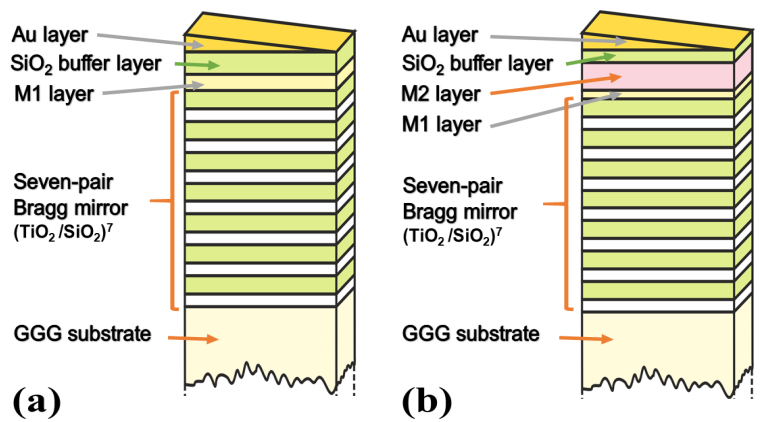

(b)

Fig. 1. Schematic diagram of synthesized structures No. 1 (a) and No. 2 (b).

\subsection{Modelling}

These structures were modelled to form a TPP mode at the center of photonic band gab (PBG) of BM. The

\footnotetext{
* Corresponding author: taciamikh@gmail.com
} 
parameters of the structure were previously calculated using the $4 \times 4$ transfer matrix method [18] for numerical solution of Maxwell's equations. The components of permittivity tensors of layers obtained by fitting the optical and magneto-optical spectra of previously synthesized single-layer films and magnetophotonic crystals were taken into account [15-17]. The same components of permittivity tensor were used for $\mathrm{Au}$ coating with thicknesses in the range from 0 to $70 \mathrm{~nm}$ [15]. The following thicknesses were used in presented in section 3 calculations of the spectra of single BM, BM with magnetic layers and structures without and with the top Au coating:

$h_{\mathrm{TiO} 2}=73 \mathrm{~nm}$ and $h_{\mathrm{SiO} 2}=115 \mathrm{~nm}$ for BM layers;

$h_{\mathrm{M} 1}=108 \mathrm{~nm}$ and $h_{\mathrm{bSiO} 2}=140 \mathrm{~nm}$ for $\mathrm{M} 1$ and $\mathrm{SiO}_{2}$ buffer layers of structure No. 1;

$h_{\mathrm{M} 1}=55 \mathrm{~nm}, h_{\mathrm{M} 2}=178 \mathrm{~nm}$ and $h_{\mathrm{bSiO} 2}=80 \mathrm{~nm}$ for M1, M2 and $\mathrm{SiO}_{2}$ buffer layers of structure No. 2 .

The PBG center of both structures is placed at $\lambda_{0}=655 \mathrm{~nm}$.

\subsection{Synthesis}

Synthesis of the structures occurred in several stages. Dielectric BM were synthesized by electron beam evaporation on hot $\left(400^{\circ} \mathrm{C}\right)$ substrate. The thickness of layers was optically controlled during deposition. Iron garnet layers were fabricated by reactive ion beam sputtering of corresponding ceramic targets in argonoxygen mixture and crystallized in the annealing process at the air. Detailed description of the methods and conditions of garnet synthesis can be found in [15-17].

$\mathrm{Au}$ thin film with a gradient of thickness $h_{\mathrm{Au}}$ from 0 to $70 \mathrm{~nm}$ along the chosen direction on the sample
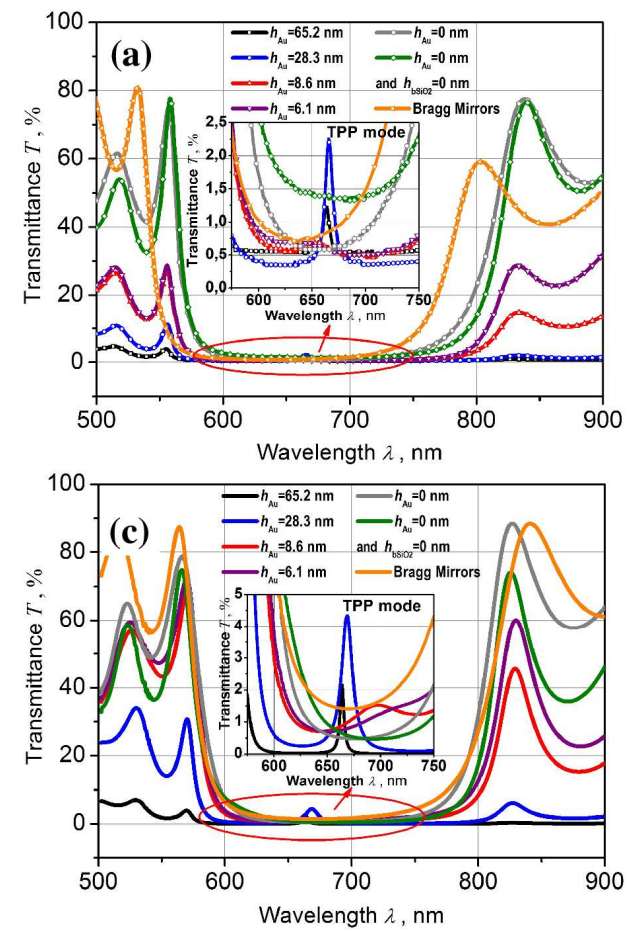

surface was deposited by thermal evaporation in vacuum using a technique described in $[19,20]$. The length of sample along the gradient of $\mathrm{Au}$ thickness was $12 \mathrm{~mm}$. Therefore, the gradient of Au thickness was $5.8 \mathrm{~nm} / \mathrm{mm}$. Garnet and $\mathrm{Au}$ thickness was determined by MII-4 microinterferometer.

Investigation of transmittance was carried out using an automated spectrophotometer KFK-3. Measurements of Faraday rotation (FR) were performed using handmade computer-control spectropolarimeter by compensation method in saturation fields. The beam aperture and the gradient of $\mathrm{Au}$ thickness at the scale of beam aperture were respectively $0.1 \mathrm{~mm}$ and $0.6 \mathrm{~nm}$.

\section{Results and discussion}

\subsection{Structure with single Bi: IG layer}

Spectral dependencies of transmittance and magnetooptical FR as a function of the thickness of $\mathrm{Au}$ coating for structure No. 1 are shown in Figure 2. The spectra of BM, BM with magnetic layer and structure without $\mathrm{Au}$ coating are also presented.

A characteristic feature of the spectra is the presence of a pronounced resonant peak, the position of which depends on the thickness of Au layer. In the calculated spectra with increasing of Au thickness $h_{\mathrm{Au}}$ from 6.1 to $65.2 \mathrm{~nm}$, the blue shift of resonance peak of TPP mode from 710 to $664 \mathrm{~nm}$ takes place. Nevertheless, the red shift of resonant mode from 640 to $664 \mathrm{~nm}$ is observed in experimental spectra for $h_{\mathrm{Au}}$ in the range from 6.1 to $30 \mathrm{~nm}$. Blue shift of resonance from 666 to $664 \mathrm{~nm}$ occurs in the experiment for $h_{\mathrm{Au}}$ in the range from 30 to
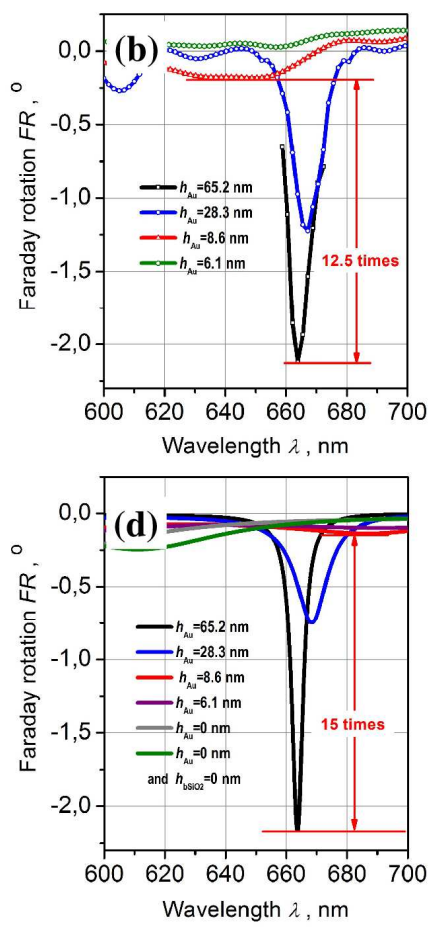

Fig. 2. Measured (a, b) and calculated (c, d) transmittance and FR spectra of synthesized seven-pair Bragg mirror, Bragg mirror with single garnet layer and TPP structure No.1 with single garnet layer as a function of the thickness of Au coating. 

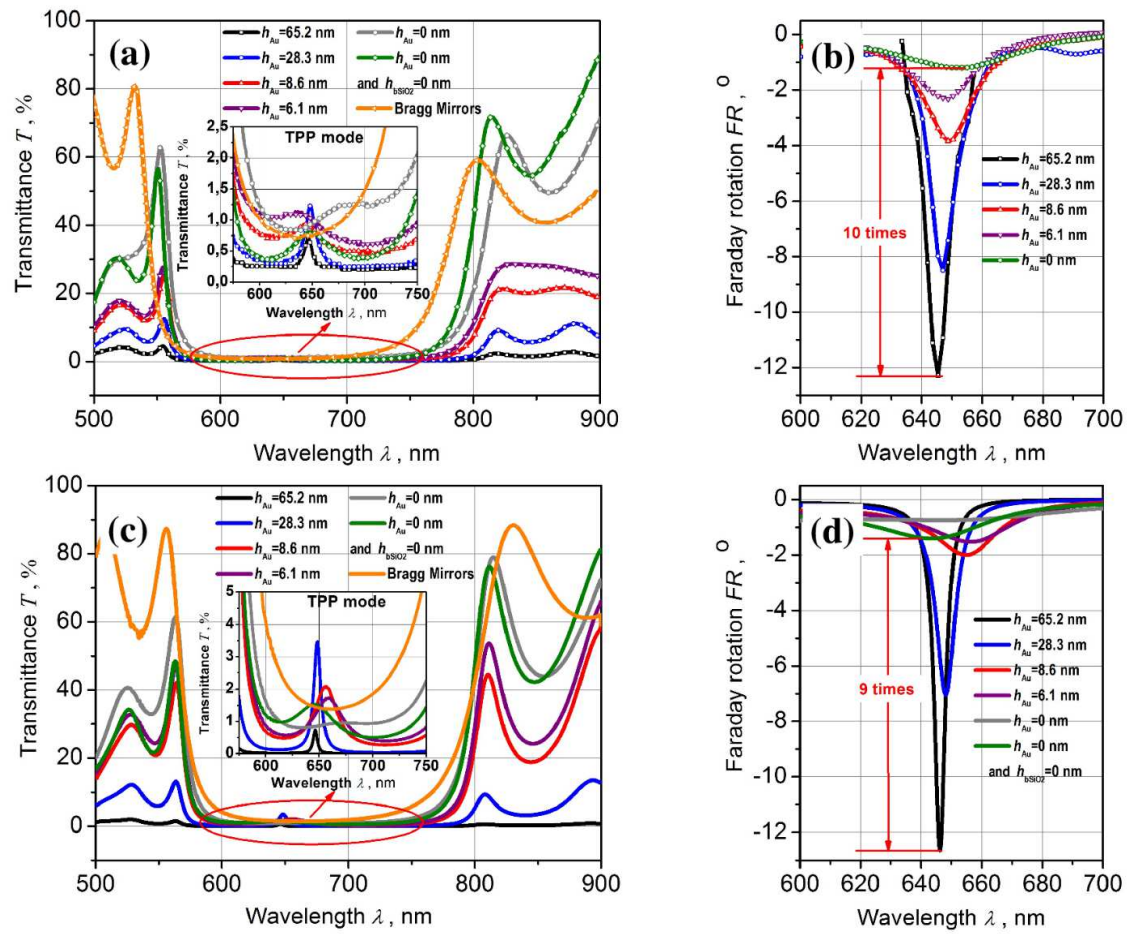

Fig. 3. Measured $(a, b)$ and calculated (c, d) transmittance and FR spectra of synthesized seven-pair Bragg mirror, Bragg mirror with double garnet layer and TPP structure No.2 with double garnet layer as a function of the thickness of Au coating.

$65.2 \mathrm{~nm}$. A reason of red shift of the mode is the changes of granularity, surface roughness and continuity of $\mathrm{Au}$ coating (structural changes) with increase of its thickness, which leads to a change of optical properties of coating. Blue shift of TPP modes is caused by the change in the coating thickness itself, since in theory we assume smooth boundaries, continuity and non-gradient thickness of $\mathrm{Au}$ layer. The surface roughness of layers, discontinuity and thickness gradient of Au coating in the beam aperture in the experiments lead to increasing of half-width of the resonant peaks.

The maximum resonant FR value are $-2.1^{\circ}$ at $664 \mathrm{~nm}$ for configuration with $h_{\mathrm{Au}}=65.2 \mathrm{~nm}$.

\subsection{Structure with double Bi: IG layer}

Measured and calculated transmittance and FR spectra of synthesized structure No. 2 are shown in Figure 3. In the spectra the shifts of TPP mode are observed analogous to the shifts in the spectra of structure No. 1. In the calculated spectra with increasing of $h_{\mathrm{Au}}$ from 0 to $65.2 \mathrm{~nm}$, the blue shift from 675 to $645 \mathrm{~nm}$ takes place. In the experiment, the red shift is from 638 to $649 \mathrm{~nm}$ for $h_{\mathrm{Au}}$ in the range from 0 to $30 \mathrm{~nm}$. Blue shift is from 649 to $645 \mathrm{~nm}$ for $h_{\mathrm{Au}}$ in the range from 30 to $65.2 \mathrm{~nm}$. Experimental and calculated spectra of structure without Au coating are identical and have the peak at $675 \mathrm{~nm}$.

The maximum resonant $\mathrm{FR}$ value are $-12.3^{\circ}$ at $645 \mathrm{~nm}$ for configuration with $h_{\mathrm{Au}}=65.2 \mathrm{~nm}$.

Calculated spatial distribution of electric field intensity in structure No. 2 with $\mathrm{Au}$ coating of different thickness, structure No. 2 without Au coating, BM with magnetic layers and single BM is shown in Figure 4.

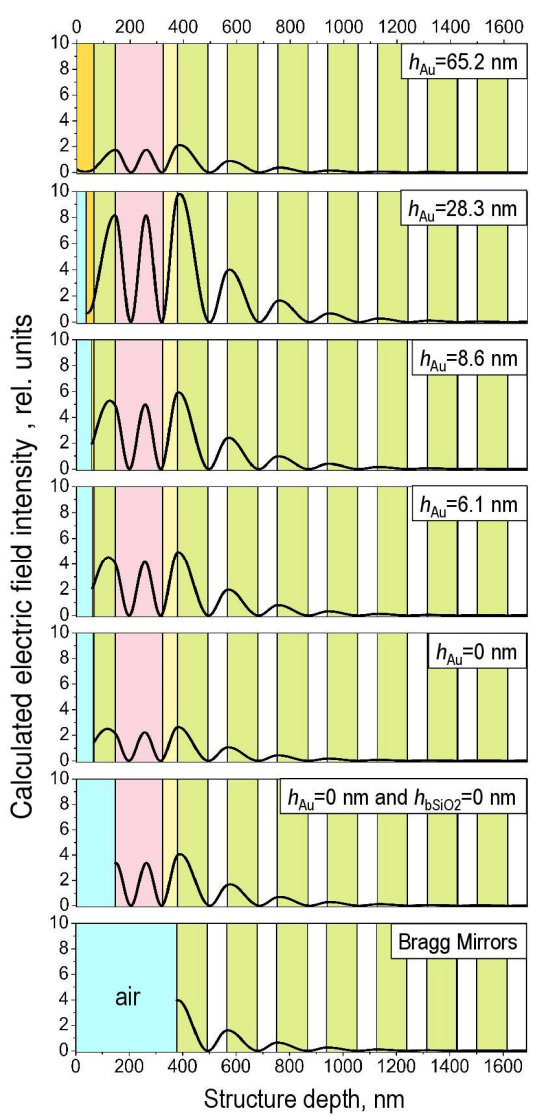

Fig. 4. Calculated spatial distribution of electric field intensity in structure No. 2 with different Au thickness, structure No. 2 without Au coating, BM with magnetic layers and single BM. 
The distribution is classical for TPP mode. Distribution in magnetic double layer is characterized by asymmetry. The maximum intensity is observed in the layers adjacent to the plasmonic coating and for configuration with thickness of Au layer of $28.3 \mathrm{~nm}$. This is in good agreement with the experimental results and indicates that a coating thickness is optimal for such type of structure. Consequently, the resonances on TPP has the maximum optical quality factor and transmittance at the vicinity of Au thickness of $30 \mathrm{~nm}$ for both structures No. 1 and No. 2 .

\section{Conclusions}

In the work, the new original magnetophotonic structures $\quad\left[\mathrm{TiO}_{2} / \mathrm{SiO}_{2}\right]^{7} / \mathrm{M} 1 / \mathrm{SiO}_{2} / \mathrm{Au}$ and $\left[\mathrm{TiO}_{2} / \mathrm{SiO}_{2}\right]^{7} / \mathrm{M} 1 / \mathrm{M} 2 / \mathrm{SiO}_{2} / \mathrm{Au}$ with $\mathrm{Bi}$-substituted iron garnets of composition $\mathrm{Bi}_{1.0} \mathrm{Y}_{0.5} \mathrm{Gd}_{1.5} \mathrm{Fe}_{4.2} \mathrm{Al}_{0.8} \mathrm{O}_{12}$ (M1) and $\mathrm{Bi}_{1.5} \mathrm{Gd}_{1.5} \mathrm{Fe}_{4.5} \mathrm{Al}_{0.5} \mathrm{O}_{12}(\mathrm{M} 2)$ and $\mathrm{Au}$ plasmonic layer were proposed and synthesized. The structures were modelled to form a TPP mode at the center of photonic band gab.

For the first time, the investigation of the features of TPP resonances for TPP structures as a function of the thickness of metal coating was done experimentally and theoretically. The resonances on TPP have the maximum optical quality factor and transmittance at the vicinity of $\mathrm{Au}$ thickness of $30 \mathrm{~nm}$. Calculations show that these configurations are optimum to form the highest intensity of electric field of light wave in the area of the magnetic layers. It was found the spectral shift of TPP mode with increasing of $\mathrm{Au}$ thickness. Spectral red and blue shifts are caused by different factors. A reason of blue shift of the mode in the experiment is the structural changes in Au coating with increase of its thickness. The red shift of TPP modes is caused by the change in the coating thickness itself.

It was achieved the maximum resonant values of FR $-2.1^{\circ}$ at $664 \mathrm{~nm}$ and $-12.3^{\circ}$ at $645 \mathrm{~nm}$ for structures with single and double garnet layers, respectively, and thickness of Au coating of $65.2 \mathrm{~nm}$.

The authors acknowledge support by the RF Ministry of Education and Science in the framework of the state task (project no. 3.7126.2017/8.9).

\section{References}

1. M. Inoue, K. Arai, T. Fujii, M. Abe, J. Appl. Phys. 85, 5768 (1999)

2. M. Inoue, A.V. Baryshev, T. Goto, S.M. Baek, S. Mito, H. Takagi, and P.B. Lim, Magnetophotonics (Springer-Verlag Berlin Heidelberg, 2013)

3. I.L. Lyubchanskii, N.N. Dadoenkova, M.I. Lyubchanskii, E.A. Shapovalov, Th. Rasing, Journal of Physics D: Applied Physics 36, R277 (2003)

4. S. Kahl and A.M. Grishin, Appl. Phys. Lett. 84, 1438 (2004)
5. S.I. Khartsev and A.M. Grishin, J. Appl. Phys. 101, 053906 (2007)

6. A.P. Vinogradov, A.V. Dorofeenko, S.G. Erokhin, M. Inoue, A.A. Lisyansky, A.M. Merzlikin and A.B. Granovsky, Phys. Rev. B 74, 045128 (2006)

7. M. Kaliteevski, I. Iorsh, S. Brand, R.A. Abram, J.M. Chamberlain, A.V. Kavokin, and I.A. Shelykh, Phys. Rev. B 76, 165415 (2007)

8. T. Goto, A.V. Dorofeenko, A.M. Merzlikin, A.V. Baryshev, A.P. Vinogradov, M. Inoue, A.A. Lisyansky and A.B. Granovsky, Physical Review Letters 101113902 (2008)

9. M. Kaliteevski, S. Brand, R.A. Abram, I. Iorsh, A.V. Kavokin and I.A. Shelykh, Applied Physics Letters 95251108 (2009)

10. A.P. Vinogradov, A.V. Dorofeenko, A.M. Merzlikin, Uspekhi Fizicheskikh Nauk 180 249 (2010)

11. R. Brückner, M. Sudzius, S.I. Hintschich, H. Fröb, V.G. Lyssenko and K. Leo, Phys. Rev. B 83033405 (2011)

12. Hai-Chun Zhou, Guang Yang, Kai Wang, Hua Long, Pei-Xiang Lu, Chin. Phys. Lett. 29, 067101 (2012)

13. B.I. Afinogenov, V.O. Bessonov, A.A. Nikulin and A.A. Fedyanin, Applied Physics Letters 103, 061112 (2013)

14. S.K. S.-U. Rahman, T. Klein, S. Klembt, J. Gutowski, D. Hommel and K. Sebald, Sci. Rep. 6, 34392 (2016)

15. T.V. Mikhailova, A.V. Karavainikov, A.N. Shaposhnikov,

V.N. Berzhansky, A.R. Prokopov and S.D. Lyashko, J. Phys.: Conf. Ser. 741, 012121 (2016)

16. V.N. Berzhansky, A.V. Karavainikov,

T.V. Mikhailova, A.N. Shaposhnikov, Yu.N. Kharchenko,

A.R. Prokopov, I.N. Lukienko, N.F. Kharchenko, J. Magn. Soc. Jpn. 36, 42 (2012)

17. V. Berzhansky, A. Shaposhnikov, A. Prokopov, A. Karavainikov, T. Mikhailova, I. Lukienko, Yu. Kharchenko, V. Golub, O. Salyuk, V. Belotelov, JETP 123, 744 (2016)

18. C.P. Yin, T.B. Wang and H.Z. Wang, Eur. Phys. J. B 85, 104 (2012)

19. S.V. Tomilin, A.S. Yanovsky, J. of Nano- and Electronic Physics 5, 03014 (2013)

20. S.V. Tomilin, V.N. Berzhansky, A.N. Shaposhnikov, A.R Prokopov, E.T. Milyukova, A.V. Karavaynikov and O.A. Tomilina, J. Phys.: Conf. Ser. 741, 012113 (2016) 